\title{
Research on the Arrangement Design of Competitive Aerobics Complete Movements Based on Feature Extraction
}

\author{
Wei Yang*, Zixin Ma, Xin Feng, Meng Wang \\ Fenyang College of Shanxi Medical University, Fenyang, Shanxi, 032200, China \\ *corresponding author
}

Keywords: Feature Extraction, Competitive Aerobics, Complete set of Movements, Layout Design

\begin{abstract}
Competitive aerobics consists of different types of movements, such as aerobics movements and modeling, difficulty movements, coordination movements of collective events and transition and connection movements. High-level complete sets of movements require a complete display of a whole. The connection of each movement, the conversion of each spatial position, and even the preparation of each difficult movement require that they must be connected with each other by dynamic and vivid and novel methods. The design of complete sets of competitive aerobics movements is the embodiment of the participating teams' wisdom contest and cultural exchange according to the rules. It is no longer merely a confrontation between physical fitness and skills. The arrangement should embody the beauty of action, structure and music, and conform to the requirements of the rules. Based on feature extraction, this paper focuses on the research and analysis of the function and design of transition and connection movements in the complete set of competitive aerobics movements in order to provide valuable reference for improving the level of competitive aerobics in China.
\end{abstract}

\section{Introduction}

The arrangement of complete sets of movements is a necessary condition for participating in the competition. The quality of creation directly affects the performance effect and competition results of athletes [1]. Under the guidance of the new rules, it is required that the movement should be ornamental and artistic in its movement arrangement. In terms of movement difficulty, it should start with novelty and high difficulty. But also can further develop students' coordination, rhythm and expressive force, and improve students' learning interest and learning effect [2]. Contents of competitive aerobics (seven basic steps and special contents, transition and connection, space); Performance (expressiveness, harmony). The highest score is 10 points, and the artistic performance of the set of movements will be reduced [3]. The design of complete sets of competitive aerobics movements is the embodiment of the participating teams' wisdom contest and cultural exchange according to the rules. It is no longer merely a confrontation between physical fitness and skills. The arrangement should embody the beauty of action, structure and music, and conform to the requirements of the rules. After the introduction of the new rules, there are not many materials to study the arrangement of competitive aerobics in China. Based on feature extraction, this paper focuses on the research and analysis of the function and design of transition and connection movements in the complete set of competitive aerobics movements, revealing the arrangement rules and features of difficult movements in the complete set of competitive aerobics movements, and providing valuable reference for improving the level of competitive aerobics in China.

\section{Relevant Principles of Complete Set of Movements Arrangement in Competitive Aerobics Collective Events}

\subsection{Innovation Principle}

Rules are rules, which are the basis for judges to score and for athletes to arrange complete sets of actions. If the rules are ignored in the arrangement, unnecessary points will be deducted. When 
arranging complete sets of actions, a single mode cannot be adopted. All the connecting and transitional movements, including the preparation of difficult movements, must really show the characteristics of aerobics [4]. The quality of sports directly affects athletes' skills and achievements, but different athletes have different sports qualities. It is a process in which individual movements are regularly organized in series to create competitive aerobics routines. The transition and connection movements naturally and skillfully connect various types of movements, not only showing their unique artistry, but also the design of the transition and connection movements themselves is extremely artistic. A complicated creative process based on competition rules, its aesthetic components and coaches' innovative thinking [5].

\subsection{Scientific Principle}

Competitive aerobics consists of different types of movements, such as aerobics movements and modeling, difficulty movements, coordination movements of collective events and transition and connection movements. When selecting athletes to form a three-person team, attention must be paid to the comprehensiveness of sports quality. The difference in sports quality cannot be too large, otherwise athletes with defects in sports quality will directly affect the level of the overall team. This is a reflection of the objective law of the editing process and a summary of the editing practice experience, which is of universal guiding significance to editing [6]. The transition and connection movements can well show the artistic style of complete sets of movements, with the artistic effect of icing on the cake, and can also reflect the variability of movements, making people feel that movements emerge in endlessly.

\subsection{Competitive Principle}

The arrangement of movements cannot be divorced from the actual situation of athletes' competitive ability. The level of competitive ability of athletes is the main basis for determining the style, difficulty, structure and requirements of their movements arrangement. High-level complete sets of movements require a complete display of a whole. The connection of each movement, the conversion of each spatial position, and even the preparation of each difficult movement require that they must be connected with each other by dynamic and vivid and novel methods. The style and characteristics of the complete set of movements are displayed through the design of the athletes' core movements and the changes of music rhythm and melody, which are the integral characteristics of the athletes' internal passion and performance skills. It reflects the collective, creative, complementary and intelligent communication process between subject and object. The spatial transformation function of transition and connection actions is relatively improved. Transition and connection actions have become an important way of spatial transformation.

\section{Elements of Arrangement of Complete Set of Actions}

\subsection{Action Element}

With the implementation of the new rules, it is required that the difficulty arrangement of movements be more balanced. In the complete set of movements, the choice of difficult movements tends to be diversified, especially for some innovative difficult movements. No matter the champion or the Chinese player, the asymmetrical movements of the upper limbs are far more than the symmetrical movements. Exercises must include many diversified combinations of steps and arm movements. Basic steps are used to deduce recognizable and continuous complex combinations with high body coordination.

In this paper, Hog3D method is used to extract local region features. The specific feature extraction process is shown in Figure 1 [7]. 


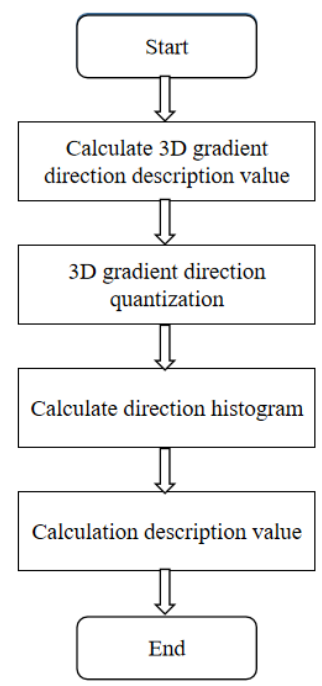

Figure 1 Hog3D aerobics movement feature extraction process

Building a feature partition model for extracting feature points of body edge contour, realizing visual penetration by adopting a bright spot model diffraction method, searching technical characteristics of aerobics athletes, and the scattering model expression of athletes' action images is [8]:

$$
S\left(r_{k}\right)=\sum_{r_{k} \neq r_{i}} w\left(r_{i}\right) D_{r}\left(r_{k}, r_{i}\right)
$$

In the formula, $w$ is the direction of the filter, $r_{i}$ is the standard deviation of Gaussian function, and in the visual feature acquisition channel, $D_{r}$ is the spot intensity of the infrared remote sensing feature transformed along with the distribution of motion.

The world coordinate systems A and B are constructed, and when the coordinate systems A and $\mathrm{B}$ have the same orientation and different origins, inverse kinematics solution of human body structure is carried out, and the view switching motion equation of multi-contour three-dimensional model visual modeling of aerobics athletes is obtained as follows:

$$
D_{\mathrm{j}}=\sum_{i=1}^{n}\left(H_{P} \times V_{p}\right)
$$

According to the multi-contour 3D model visual modeling viewpoint switching motion equation constructed above, the edge contour feature detection result is obtained as follows:

$$
S_{\mathrm{j}}=\frac{1}{\sum_{i=1}^{n}\left(S_{r}\right)},\left(0<\left(S_{\mathrm{j}}\right) \leq 1\right)
$$

Through the above analysis, the feature extraction of aerobics body is realized, which provides an accurate data basis for the design of this system.

Whether the design of transition and connection actions is reasonable or not directly affects the completion quality of difficult actions, modeling and coordination. People's perception of things around us is at the initial stage of understanding things. We not only need to observe the external appearance of things carefully, but also have the ability to have insight into the inner nature of things. The value of an athlete's set of movements in a competition and whether he can get a high score depend to a large extent on whether the difficult movements of the set of movements are novel and unique. In a set of movements, the referee should be able to clearly recognize at least one rhythm change, even if the music itself has no rhythm change. From this, we can see that the quality of the choreography directly affects the result of the competition. A good choreography should focus on novelty and uniqueness in order to have artistic appeal and arouse the interest of the audience and the judges. 


\subsection{Musical Elements}

Whether the music selection is reasonable or not has an important influence on the potential stimulation of athletes. Therefore, compared with foreign athletes, the music selected by our country in the competition has received extensive attention. The symmetrical rhythm of the phrase creates a natural and good condition for the symmetry of the movement, which makes the rhythm of the movement consistent with the music melody and helps the movement find the rhythm and lasting appeal of the body. However, "difficulty" cannot be considered as the only winning factor, and more attention should be paid to the diversification of movements and the addition of "difficulty" in movement connection. New technologies, new difficulties and new connections should be available. Athletes should complete different kinds of difficult movements of flexibility, at least one for each of the left and right legs, or one to show the flexibility of both sides of the body [9]; The style of the set of movements must be consistent with the concept of music (melody, beat, remake, segment). The complete set of movements must express the concept of music, the beat must be clear, and the movements must be prominent in the remake. In the whole creation process should be in a dominant position. We need to have excellent aerobics professional knowledge and understand aerobics related knowledge, such as aerobics rules, other related sports, arts and so on.

\subsection{Time and Space Factors}

In arranging complete sets of movements, it is very important to make effective use of the competition venue. For aerobics, it should not be limited to narrow venues, but should make maximum use of the competition venue. The diversity of the transition from air to ground and their mutual conversion is stipulated as follows: athletes will be penalized if they repeat exactly the same movement every time they switch from one plane to another. Generally speaking, human body movements occupy different levels in space: high, medium and low. The various movements made by the athletes in the upright state are hollow, the various jumping movements are high altitude and the ground movements are low altitude.

Understanding the different spaces of human body movement is helpful for rational use of each space, making the whole set of movements more varied and more prominent in aesthetic characteristics (see Table 1).

Table 1 Aesthetic features of different lines

\begin{tabular}{|c|c|}
\hline Type & Aesthetic features \\
\hline Straight line & Strong; Tall and straight \\
\hline Slash & Continuation; Promotion; depth \\
\hline Horizontal line & Ease; Stable; Quiet \\
\hline Curve & Lively; Jumping; Swimming; Unstable \\
\hline
\end{tabular}

High-difficulty movements must be guaranteed by high quality and high stability. At critical moments, one would rather give up a little difficulty to guarantee quality. When the difficulty approaches, the movement quality is the key to scoring. Diversity of difficulty movements: points will be deducted if two identical difficulty movements occur in the set of movements. Without this prerequisite, things cannot move forward. Active participation spirit is the catalyst for calisthenics development. Attention should also be paid to the diversified arrangement of arm movements, such as arm movement height, angle, movement plane, movement amplitude, limb length, movement speed, rhythm change and asymmetry. The use of the three spaces (ground, standing and air) must be interspersed with each other in a dynamic, smooth and flowing manner.

\section{Countermeasures for Arrangement and Design of Competitive Aerobics Complete Set of Movements}

\subsection{New Design Difficulty and Combination Difficulty}

The whole set of movements of competitive aerobics should be arranged according to rules and according to athletes' abilities and characteristics. The competitive ability possessed by competitive 
calisthenics athletes is mainly manifested in the accurate completion of each beat in the process of completing the complicated combination of exercises. However, each team still has a certain distance to show a set of creative actions properly. Not only should attention be paid to the selection of various types of movements, but also to the application of various types of movements, so as to enrich the complete set of movements, so that all parts of the body can be exercised, and at the same time conform to the requirements of the rules, which is helpful to obtain a higher score. Stability, accuracy and neatness are the standards of action quality. The stability of action is greatly affected by accuracy and is also closely related to the degree of action completion. Even the preparation of every difficult movement requires that it must be connected with each other in a dynamic and vivid and novel way. It must change the space of the action, and the connecting action connects two different contents. The action itself does not allow the space to change and cannot be repeated. The transition and connection movements are relatively short and flexible, and easy to connect with difficult movements, lifting and dynamic coordination, and starting and ending movements.

\subsection{Strengthen the Complexity and Creativity of the Choreography}

The combination of movements and the choice of difficult movements must be well integrated and balanced in different spaces and cannot be concentrated on one plane. Innovation is one of the key factors that determine the achievement of competitive aerobics in competition. Only innovative actions can embody higher value. Through beautiful images, beautiful movements, beautiful lines, beautiful dances, beautiful patterns and beautiful melodies, people can enjoy beauty. To cultivate athletes' good mentality. For each athlete, it is necessary to improve their comprehensive physical quality, train athletes to have a stable competitive mentality, and give full play to the best economic conditions under the guidance of the new rules. The arrangement of the set of movements must have three lifts, which can include the opening and ending, but it must show different body shapes and formations, and must be integrated with the music completely and smoothly. The editing of a piece of music must show natural emotion and smooth conversion. If you want to add dynamic effect to a complete set of music, you must have matching actions to enhance the complete set of effect. In the process of exercise, make reasonable use of physical strength, under the premise of perfect display of movement, make the most economic use of human energy, and avoid unnecessary consumption of human energy. The promotion of innovative consciousness, the promotion of innovative elements in the arrangement of complete sets of contents, and the rules of competitive aerobics have great impetus to the development of competitive aerobics.

\subsection{Strengthen the Artistry and Appreciation of Action Choreography}

The composition of a set of actions lies not in the action itself but in its connection. To achieve smooth and smooth combination of actions, the end of the previous action must become the beginning of the latter. At the beginning of its development, competitive aerobics shows the beauty of the human body with its perfect movements. It has extremely high appreciation. The artistry of complete sets of movements is the key to athletes' artistic scores. Music is the soul of competitive aerobics. The choice of music has a strong sense of rhythm. Disco music or rock music with strong rhythm can be selected. Sound effects can be used in the new rules, but points will be deducted if they are not used well. According to the rhythm of the music, arms, legs or other parts of the body complete the movements at different rhythms. Therefore, when arranging complete sets of movements, one should not only consider whether the movements match the musician's rhythm, but also consider whether the selected movements can express the mood of music and whether the style can reflect the characteristics of music. In the actual arrangement, considering the continuous innovation of the arrangement of difficult movements, the difficulty coefficient of competitive aerobics is improved, and its appreciation and artistry are also improved. A good use of music can help athletes fully display the perfect combination of movements and music, and improve athletes' personality and technical style. In order to grasp the correct development direction of competitive aerobics, one should not only do actions that are harmful to one's body in pursuit of difficulty, but also pay attention to avoiding illegal actions stipulated in the rules. 


\section{Conclusion}

The revision of the rules of competitive calisthenics guides the direction of calisthenics. In the arrangement of complete sets of movements, more and more emphasis is placed on the innovation and diversity of movements such as gymnastics, difficult movements, transitional connection and lifting. The best set of movements for competitive aerobics group events cannot be arranged without the characteristics of the athletes themselves and should be arranged to the greatest extent to suit the athletes' own style. More attention should be paid to the arrangement of formation changes, and the selection and use should be more diversified, especially the training of formation changes diversity. It requires athletes to carry out hard training under the guidance of coaches. While continuously improving athletes' sports skills, they should also strengthen psychological training so as to achieve ideal results through difficult, new and skillful arrangement, stability, accuracy and beauty in the competition.

\section{References}

[1] Zhang Xinjiao. Research on the arrangement of the complete set of movements for collective events of competitive aerobics under the guidance of rules. Contemporary Sports Science and Technology, vol. 6, no. 10, pp. 142-143, 2016.

[2] Jiang Minghuan. Analysis and Research on the Artistic Arrangement of Competitive Aerobics and Five People in the Background of New Rules. Sports Fashion, no. 9, pp. 286-286, 2019.

[3] Wang Hui, Zhou Jianshe. An Analysis of the Application and Development of Competitive Aerobics Arrangement Art. Sports, no. 016, pp. 26-27,97, 2016.

[4] Feng Tingting. Research on the success rate of the difficulty named after Liusingen in the aerobics package_— Taking the 2012 World Championships as an example. Youth Sports, no. 12, pp. 61-63, 2015.

[5] Yang Yunxia. Artistic comparative analysis of the complete set of movements in the women 's singles event of the 14th World Aerobics Championship. Fujian Sports Science and Technology, vol. 36, no. 06, pp. 40-42, 2017.

[6] Guan Luohong, Cheng Zhengtao. Discussion on the application of the "building block principle” in the aerobics course reform. Stationery and Sport Supplies and Science and Technology, no. 18, pp. 52-52, 2015.

[7] Li Tihao. Research on the arrangement of difficult movements in competitive aerobics. Stationery and Sports Supplies and Technology, no. 5, pp. 30-31, 2019.

[8] Sun Tao. Research on the formation characteristics of the five-person competitive aerobics project. Science Teaching Journal-Electronic Edition, no. 2, pp. 92-92, 2015.

[9] Zhang Zhuo, Luo Tengxiang. Comparative analysis of domestic and international competitive aerobics women's single difficulty movement technique and its development trend. Sports, no. 11, pp. 15-18, 2015. 